\title{
TRATTI DEL PARLATO NELLE E-MAIL D'AFFARI IN LINGUA ITALIANA SCAMBIATE TRA PARTNER ITALIANI E SLOVENI
}

\section{INTRODUZIONE 1}

Nell'ambito dello studio dell'italiano per gli affari (L 2) per futuri economisti, alla $\mathrm{EF}^{2}$ vengono insegnate le competenze tanto nel campo della comunicazione parlata (riunioni, negoziazioni, conversazioni telefoniche) quanto nel campo di quella scritta (lettera cartacea, fax, e-mail). Per il versante scritto, i libri di testo usati e i manuali disponibili offrono all'insegnante e allo studente abbondante materiale per la redazione di lettere tradizionali e di fax, mentre, i modelli per le e-mail scarseggiano. Pertanto, alcuni anni fa, dall'autrice, docente d'italiano alla detta Facoltà, è stata intrapresa una ricerca volta a verificare i tratti distintivi della comunicazione per posta elettronica nella prassi effettiva, i cui risultati sono illustrati in Lenassi (2010). Nella parte empirica è stato così raccolto e in seguito analizzato un corpus di 294 e-mail scambiate tra partner d'affari italiani e sloveni (cf. par. 1.1).

Dalle analisi è emerso che le e-mail d'affari - come rilevato da altri studiosi già per le e-mail di comunicazione quotidiana (cf. par. 2) - abbracciano testi caratterizzati da vario grado di formalità (da quelli marcatamente ufficiali fino a quelli prettamente informali) e da vari tratti diamesici (da elementi appartenenti esclusivamente alla comunicazione scritta fino a quelli tipici del discorso parlato). Ad illustrazione di quanto detto si osservino i primi tre esempi riportati. ${ }^{3}$

Gli esempi (1) e (2) rappresentano due diverse realizzazioni di uno stesso atto di operazione commerciale, cioè di un'offerta. Il primo esibisce un atteggiamento decisamente formale (assenza di formula introduttiva, espressioni ricorrenti come $d a a c$ cordi, ns migliore offerta, e chiusura stereotipata Rimaniamo a Vs completa disposizione per ogni eventuale chiarimento e con l'occasione porgiamo cordiali saluti):

\footnotetext{
* Indirizzo dell'autrice: Ekonomska fakulteta, Katedra za tuje jezike za ekonomske in poslovne vede, Kardeljeva pl. 17, 1000 Ljubljana, Slovenia. Email: nives.lenassi@ef.uni-lj.si

${ }^{1}$ Vorrei cogliere quest'occasione per porgere i miei più vivi ringraziamenti alla prof. dr. Tjaša Miklič per le preziose osservazioni e i suggerimenti relativi a una versione precedente del presente articolo.

${ }^{2}$ Facoltà di economia dell'Università di Lubiana/Ekonomska fakulteta Univerze v Ljubljani.

${ }^{3}$ Per evidenziare le singole particolarità linguistiche, le e-mail impiegate nell'articolo sono state oggetto di taluni interventi quali sottolineature e il grassetto. Per il resto i testi sono stati mantenuti in forma originale, inclusi gli eventuali errori di battitura o altro. Per il significato delle abbreviazioni, degli asterischi e l'anonimizzazione v. il par. 1.1.
} 
(1) Oggetto: Offerta

Come da accordi inviamo ns migliore offerta per i seguenti macchinari:

- Pressa $U^{* * *}$ per $\mathrm{s}^{* * *}$

USATA REVISIONATA

Euro

Rimaniamo a Vs completa disposizione per ogni eventuale chiarimento e con l'occasione porgiamo cordiali saluti

$\mathrm{R}^{*} \quad(\mathrm{O} 1)$

Il secondo, invece, è caratterizzato da tratti conversazionali e di prossimità sociale tra gli scriventi (Ciao D*, ecco, calcola che, saluti):

(2) Oggetto: LANA DI VETRO PESANTE AUTOADESIVA TAGLIATA

Ciao D*,

ecco la nostra offerta per la lana di vetro pesante autoadesiva tagliata in altezza 7 e 15 $\mathrm{mm}$ come da tuoi campioni:

$-7 \mathrm{~mm}$ : /RT (mix $7 \mathrm{~mm}$ x $50 \mathrm{mt}$ ) min. da acquistare $214 \mathrm{RT}$

- $15 \mathrm{~mm}$ : /RT (mix $15 \mathrm{~mm}$ x $50 \mathrm{mt}$ ) min. da acquistare $100 \mathrm{RT}$

Calcola che ad ogni rotolo $\mathrm{H}$ 150, al prezzo della lana di vetro, viene aggiunto un costo di taglio di

\section{Saluti}

$S^{*} S^{* *}$

(O 29)

L'es. (3), infine, con i propri tratti lessicali e le "traduzioni" grafiche (puntini di sospensione, da assumere forse come segnale di pausa indicante che il tema non è del tutto esaurito) sembra mimare una comunicazione per telefono (lasciata alla segreteria telefonica):

(3) Oggetto: Re: Contratto

Cara I*,

grazie come sempre per la tua tempestivita'.

Bene, attenderemo la copia del contratto, poi vedremo.

Quanto a $\mathrm{R}^{*}$, interessante...

Ci sentiamo appena rivero,4

Con l'augurio che il lavoro non sia troppo oberante...

un abbraccio,

A* (AP 19)

Data una notevole presenza, nella comunicazione d'affari analizzata, di un registro informale, esternato anche nel ricorso alla modalità parlata, sorge a questo punto la domanda quali elementi del parlato (cf. par. 3) vi siano presenti e in quali situazioni

\footnotetext{
${ }^{4}$ Errore di battitura al posto di rivedro'.
} 
comunicative nei testi pur sempre caratterizzati - rispetto alla corrispondenza privata - da una relativa istituzionalità.

\subsection{Dati relativi al corpus}

La raccolta dei testi di posta elettronica è stata dall'autrice effettuata, tra il 2005 e il 2010, in varie istituzioni e aziende slovene che con l'Italia intrattengono rapporti d'affari in lingua italiana. ${ }^{5}$

Dato che da elementi contenuti nelle e-mail eventuali segreti professionali potevano essere riconosciuti, a raccolta ottenuta si è proceduto all'anonimizzazione. ${ }^{6}$ Per tutelare i dati relativi alle aziende e alle persone ci si è serviti dei codici nel modo seguente: $A^{*}$ per nome di una persona, $A^{* *}$ per cognome di una persona, $A^{* * *}$ per nome dell'azienda/nome del marchio, $A^{* * * *}$ per nome geografico. La combinazione alfanumerica in calce al singolo esempio riportato si riferisce al singolo esemplare della collezione archiviata dall'autrice.

Il corpus ottenuto consta di 193 testi prodotti da madrelingua e di 101 testi prodotti da non madrelingua (sloveni). Gli esemplari rientrano in varie tipologie testuali (offerta, risposta all'offerta, reclamo, risposta al reclamo, notifica dell'invio dell'allegato, ecc.) e contengono da 5 fino a 307 parole a testo. ${ }^{7}$

\section{CARATTERISTICHE LINGUISTICHE E TESTUALI DELLE E-MAIL}

Non esiste praticamente saggio sulla posta elettronica in cui non siano chiamati in causa il parlato, l'imitazione del parlato, la comunicazione faccia a faccia, l'oralità, le forme di comunicazione orale, la dialogicità, la colloquialità, ecc.

Trattando, infatti, le caratteristiche del messaggio elettronico in italiano, ${ }^{8}$ vari autori sono concordi circa il suo carattere ibrido, non facilmente collocabile sulla linea diamesica tra la comunicazione scritta e quella parlata (cf. Antonelli 2007: 187; Fiorentino 2002: 187; Fiorentino 2004: 84-85; Fiorentino 2008: 98-99; Lorenzetti/Schirru 2006: 94; Peticca 2005: 97). ${ }^{9}$

La Fiorentino (2008: 92), confrontando la struttura dell'e-mail del suo corpus con il modello prototipico della lettera ordinaria - caratterizzata da una parte iniziale con la data e il luogo di emissione; una formula di apertura e saluti (con talvolta il nome del destinatario); una parte centrale che contiene il motivo della scrittura; la parte finale e la firma del mittente - constata che la maggior parte dei messaggi elettronici tende ad

\footnotetext{
${ }^{5}$ Ringrazio qui i rappresentanti delle aziende e dei vari istituti, che a scopi di ricerca, hanno voluto gentilmente concedere l'uso della propria corrispondenza.

${ }^{6}$ Mentre in una piccola parte della posta gli estremi erano stati cancellati già dalle aziende stesse, prima della consegna/cessione dei testi, la sostituzione necessaria nella maggior parte dei materiali è stata dall'autrice effettuata in seguito.

${ }^{7}$ Alla stregua di altre trattazioni sulla posta elettronica, nel presente contributo viene normalmente riportato solo il corpo centrale di ogni singola e-mail. Per eventuali ulteriori informazioni si possono consultare i testi del corpus nella collezione conservata in forma autentica.

${ }^{8}$ Per l'inglese, cf. Baron 1998; Crystal 2001, ecc.

${ }^{9}$ Sulla problematica delimitazione parlato vs. scritto cf. Nencioni 1983.
} 
essere incompleta rispetto alla struttura sopra menzionata, e soprattutto più breve (fino a ridursi ad un mero saluto): "Data la sua brevità e viste anche le diverse funzioni comunicative di un messaggio, si deve concludere che la posta elettronica può sostituire forme di comunicazione anche orali: una telefonata, un messaggio lasciato ad una segreteria telefonica, un fax." (ibid.). Dal punto di vista delle caratteristiche morfologiche e sintattiche la lingua nelle e-mail è giudicata piuttosto "corretta e si può qualificare come italiano dell'uso medio (secondo una ben nota definizione di Sabatini 1985)" (ibid.: 76). ${ }^{10}$ La Fiorentino constata il variare del registro in dipendenza dal destinatario, dal contenuto, dallo scopo, come in qualunque tipo di comunicazione (ibid.). ${ }^{11}$

Antonelli (2007: 148, 149), riguardo alle caratteristiche delle nuove forme di comunicazione, individua tutta una serie di strategie espressive che mirano a ridurre la distanza comunicativa tra i due interlocutori. Secondo questi, diversi fenomeni sono riconducibili a tre fattori principali: a) alla dialogicità; b) alla carenza di tempo a disposizione e alla scarsa pianificazione, e c) al tono quasi sempre informale della comunicazione. Così al primo gruppo appartengono i saluti e le altre formule che servono ad avviare la conversazione e l'alta frequenza dei vocativi; i segnali discorsivi (danno al testo un andamento parlato) e l'uso degli emoticons, cioè faccine ottenute con caratteri della tastiera per aggiungere chiavi di interpretazione alle frasi (cf. Fiorentino 2008: 78); alcuni costrutti sintattici che tendono a mettere in rilevo un particolare tema (dislocazione a sinistra o a destra) e un alto grado di implicitezza (determinata dalle presupposizioni comuni ai due interlocutori). Alla carenza di tempo e alla scarsa pianificazione sono ascrivibili la sintassi poco complessa (che può arrivare fino alla giustapposizione) delle frasi e il raro uso di elementi connettivi. Al tono quasi sempre informale, infine, va attribuito l'impiego di un lessico colloquiale con eventuale turpiloquio e modi regionali e dialettali. Secondo Antonelli, fra queste strategie vi sono anche quelle basate sull'imitazione (o meglio, simulazione) dell'oralità, soprattutto la punteggiatura enfatica che sembra voler rendere una intonazione concitata (ibid.: 153).

\section{ELEMENTI DI LINGUA PARLATA NEL CORPUS DELLE E-MAIL D’AFFARI}

$\mathrm{Si}$ è visto, quindi, che nella posta elettronica in generale, dal carattere spesso non impegnativo, una forte presenza del parlato è data per scontata. Le analisi della posta elettronica nel nostro corpus dimostrano che anche questa comunicazione, nonostante sia di norma relativamente istituzionalizzata, permette il ricorso ai singoli ele-

\footnotetext{
${ }^{10}$ Sorvoliamo qui sulle notorie caratteristiche della comunicazione giovanile (come ad es. grafie simboliche) messe in risalto dalla pubblicistica che, contrariamente alle apparenze, sono di estensione assai limitata (cf. Antonelli 2007: 152, 153).

${ }^{11}$ Per i fattori che incidono sulla produzione dei messaggi e-mail, cf. anche Nidorfer Šiškovič (2010: 60). Sulla base del lavoro di Mallon e Oppenheim (2002), che elencano fattori come età, sesso e grado d'istruzione dello scrivente, nonché l'ambiente, lo scopo del messaggio, il tempo a disposizione, l'opinione dello scrivente sull'adeguatezza del medium e sulla sua affidabilità, i rapporti tra i comunicatori e l'alfabetizzazione elettronica, l'autrice aggiunge anche fattori come la cultura d'azienda e la padronanza di vari generi testuali da parte dello scrivente.
} 
menti generalmente ascrivibili al parlato. Ciò avviene di regola nelle situazioni in cui sono soddisfatti soprattutto i seguenti parametri: rapporto simmetrico o asimmetrico di dominanza, conoscenza personale dei comunicatori e scarso tempo a disposizione.

Vediamo a questo punto le concrete spie di lingua parlata individuate nei testi analizzati. ${ }^{12}$ Affrontiamo per prima la costruzione testuale e le sue regolarità. Un tratto che avvicina alcuni messaggi e-mail alla conversazione è il suo carattere dialogico (cioè la vicinanza temporale dei singoli turni e i rapporti e mezzi di referenza che li uniscono, cf. Fiorentino 2008: 95). Così nella sequenza di e-mail (4), (5) e (6) ${ }^{13}$ sono bene riconoscibili sia la dialogicità (si osservi del resto, nell'indicazione dell'ora in (5) e in (6), la vicinanza temporale tra le produzioni testuali) sia la referenza implicita nel secondo e nel terzo testo. Mentre il primo testo ${ }^{14}$ è semanticamente autosufficiente, gli altri due, senza la conoscenza del contenuto dei messaggi precedenti, non risulterebbero comprensibili: ${ }^{15}$

(4) Sent: Tuesday, December 04, 2007 11:44 AM

Subject: Promotional sales

Dear Distributors, see the attachments and let me have your comments.

Best regards

$\mathrm{I}^{* * *} \mathrm{Srl}$

$\mathrm{M}^{*} \mathrm{R}^{* *}$

(5) Sent: Tuesday, December 04, 2007 3:00 PM

Subject: ordine

Ciao $m^{*}$,

Allora nel ordine mi puoi dare (christmas sale)

$-2 \mathrm{x} \mathrm{c}^{* * *} 6891$ con $\mathrm{m}^{* * *}$ (6975 e 6973)

$-1 \mathrm{x} \mathrm{S}^{* * *} 6845$ con $\mathrm{m}^{* * *}(6972)$

${ }^{12} \mathrm{La}$ discussione si concentrerà soprattutto su fenomeni contenuti nelle e-mail scritte dai madrelingua, la cui produzione potrà in seguito servire da modello ai parlanti non nativi. Data, infatti, la forte oscillazione nella padronanza linguistica dei non madrelingua, una presentazione adeguata relativa ai fenomeni in questione nei loro testi italiani, peraltro molto interessanti, per limiti di spazio qui non sarebbe possibile. La problematica sarà, invece, discussa in un'altra sede, dedicata a vari aspetti dell'interferenza e alla didattica. È stato, però, sporadicamente incluso qualche caso di particolare rilievo.

${ }^{13}$ L'e-mail (IM 40) in (5) è stata redatta da un non madrelingua.

${ }^{14}$ Il testo è eccezionalmente in inglese, in quanto lo scrivente si rivolge ai clienti presenti in vari paesi. Secondo quanto riferito da alcuni informatori, molte società italiane operanti a livello internazionale comunicano con i propri clienti anche o solo in lingua inglese.

${ }^{15} \mathrm{Cf}$. Antonelli (2007: 149) il quale osserva che i testi di un serrato scambio sono caratterizzati da, "un alto grado di implicitezza che si fonda sulle presupposizioni legate al contenuto dei messaggi precedenti o a informazioni note a tutti e due gli interlocutori." 
Ti pregherei di mandarmi la fattura al piu presto, chosi la pagho subito.

Saluti

$S^{*}$

(IM 40)

(6) Sent: Tuesday, December 04, 2007 3:16 PM

Subject: Re: ordine

Scusami forse non era chiaro ma il prezzo speciale e' per un acquisto minimo di 10 pezzi.

Ciao

$\mathrm{M}^{*}$

(IM 41)

A livello lessicale, una spia dell'osmosi tra la comunicazione parlata (con le attività di dire e sentire) e quella scritta (con le attività di scrivere e di leggere), nell'ambito delle e-mail analizzate, è rappresentata dalla funzione di iperonimo dei verbi dire ${ }^{16}$ e sentire. Così l'autore dell'e-mail (7) si riferisce alla propria lettura del messaggio di I* come se questo fosse stato pronunciato, per cui si serve del verbo sentire:

(7) Cara I*, che bello sentirti! (AP 15)

Il verbo dire in sostituzione del verbo scrivere è usato, invece, nei seguenti brani: ${ }^{17}$

(8) Mi hanno risposto dicendomi (vedi sotto) che avete gia' contatti [...] (EE 35)

(9) Non mi hai detto la quantita' dei C*. (IM 18) $)^{18}$

(10) Detto ciò torniamo al ns lavoro. $\quad$ (IN 1)

A livello morfosintattico, un ulteriore tratto del parlato nelle e-mail commerciali si potrebbe riconoscere nell'uso del connettivo che così:

${ }^{16}$ È chiaro che la presenza del verbo dire di per sé non è un riflesso del discorso parlato. Diversi modelli prototipici di lettera commerciale (appartenente al registro burocratico) pullulano di occorrenze del verbo al participio passato, usato in funzione anaforica: detti prezzi, detto carico, detta scelta ecc.

${ }^{17} \mathrm{Ci}$ sono, però, dei casi in cui non è possibile stabilire se il verbo dire sia usato come iperonimo per scrivere, o si riferisca invece a una comunicazione effettivamente parlata (ad es. ad una previa telefonata o ad una conversazione faccia a faccia). Il contesto spesso non fornisce informazioni sufficienti:

- [...] ti chiedo anche di fare un controllo su $T^{* * *}$ che ci dice di avere delle fatture scadute che ancora non sono state pagate. (EE 34)

- $D^{*}$ mi $h$ detto che $H^{* * *}$ ha mandato un email in luglio: abbiamo controllato ma non abbiamo niente. (O 9)

- Come detto al tuo collega $B^{*}[\ldots](\mathrm{O} 41)$

${ }^{18} \mathrm{Il}$ verbo dire dell'es. (9) rimanda ai messaggi ricevuti precedentemente, dove la quantità a cui lo scrivente si riferisce non figurava. 
(11) fatemi sapere quando viene che così preparo la fattura. (S 11)

Pure al registro parlato è ascrivibile l'uso delle frasi interrogative introdotte dalle congiunzioni e e $\mathbf{m a}:^{19}$

(12) $\mathrm{D}^{*}$ Ma la richiesta è la stessa dello scorso anno?

Si confronti Fasulo (2009: 31, 32), che nella variante con il connettivo $e$ vede un ritorno all'agenda. Nel nostro corpus ne troviamo un esempio in un messaggio dal registro informale:

(13) Cara I*, che bello sentirti! E quali liete notizie... Grazie! (AP 15)

Tipico della lingua parlata è pure il ricorso alla dislocazione a sinistra, dettato dalle esigenze della disposizione TEMA vs. REMA, con la successione risultante NOME OGGETTO + PROFORMA OGGETTO + VERBO (cf. anche Antonelli 2007: 149):

(14) vedo che gli $S^{* * *} \underline{\text { li stai vendendo.... (IM 13) }}$

(15) la sostituzione del quadrante ve l'ho passata in garanzia (IM 32)

Nel materiale analizzato appare anche il seguente costrutto tipico del parlato, in cui la proforma atona è rafforzata da quella tonica:

(16) basta che ci mandi a noi (IS 3)

(17) $\underline{\mathrm{Mi}}$ fa davvero piacere anche a me. (AP 3)

Anche i seguenti segnali discorsivi (per il fenomeno cf. Bazzanella 2006: 456) ricordano in modo decisivo una comunicazione orale:

(18) p.s. bene vedo che gli $S^{* * *}$ li stai vendendo .... (IM 13)

(19) Ciao I*, oh finalmente in stampa! (AP 5)

(20) Beh sì certo aspettiamo... (AP 9)

(21) ok per il giorno 16 (S 13)

Nella conversazione orale, il passaggio ad una nuova tematica o l'interruzione di quella attuale, quando l'autore non la senta come del tutto esaurita, vengono spesso segnalati dal connettivo del resto; nella comunicazione scritta, invece, questo fenomeno può essere realizzato tramite l'impiego dei punti di sospensione (v. anche l'es. (3)). ${ }^{20}$

${ }^{19}$ Le frasi interrogative introdotte da ma potrebbero svolgere anche la funzione "di protesta" (cfr. Fasullo 2009: 38, 39). Di tale qualità sarebbe il seguente brano, tratto da un'e-mail di un non madrelingua:

- Ho ancora due orologi da riparare (un $\mathrm{H}^{* * *}$ e un $\mathrm{C}^{* * *}$ ). Ma e nomale che ci sono tanti malfunzionamenti specialmente sui $\mathrm{H}^{* * *}$ ? Tanti clienti ci informano [...] (IM 28)

${ }^{20}$ Una funzione simile è svolta, tanto nella lingua parlata quanto nelle e-mail quotidiane, dal segnale discorsivo comunque. Cf. Fiorentino (2008: 81). 
Se di regola i punti sono tre, nei testi analizzati questa norma ortografica non viene sempre rispettata, come testimoniano i seguenti brani:21

(22) Cara I*, come stai? Spero che stiate in vacanza...

(23) [...] quindi, se anche mettiamo in produzione un'altra volta lo stesso art. poi veniamo ancora scartati per i problemi tecnici.... Cosa facciamo?? Fammi sapere... (O 5)

(24) p.s. bene vedo che gli $\mathrm{S}^{* * *}$ li stai vendendo... (IM 13)

Un altro tratto determinato dalla natura dialogica dell'e-mail è l'uso della domanda diretta (cf. D'Achille 2006: 237). Non tutte le interrogative, però, hanno la stessa funzione: alcune vengono usate per stabilire o riprendere il contatto (Ciao I*, Come stai? (AP 4)); altre - con l'uso del verbo modale "potere" - fungono da richieste di azioni (Potete quindi inviare con la prossima consegna 5 delle vostre gabbie metalliche piene? (K 12) o Puoi verificare con lo stampista qual'e' il sottosquadra che impedisce la realizzazione dello stampo? (K 22 )); altre, infine, sono usate come richieste di informazione (Chi è il vostro riferimento commerciale in $Z^{* * *}$ ? (K 16)).

Una ulteriore spia di un grado minore di formalità nella posta elettronica, è la presenza della punteggiatura allungata ${ }^{22}$ (cf. Fiorentino 2008: 82). Nel caso dell'impiego di più punti interrogativi si tratta per lo più di una funzione pragmatica, in particolare per segnalare il bisogno dell'autore di una risposta tempestiva: ${ }^{23}$

(25) mi fai sapere cosa dicono????

(IN 1)

(26) [...] mi conferma indirizzo esatto di consegna e di fatturazione???

(27) Cosa facciamo?? (O 5)

(BD 13)

Alcuni testi contengono il punto esclamativo come segnale per suggerire atti comunicativi diversi e possono essere interpretati come una specie di traduzione grafica di varie modulazioni intonative della comunicazione orale. Nei seguenti brani la punteggiatura impiegata mette in risalto in (28) un augurio o in (29) un benevolo rimprovero:

(28) Se non conosci questo tipo di lancio stampe, posso aiutarti. Buon lavoro! (EE 30)

(29) [...] bene vedo che gli $S^{* * *}$ li stai vendendo .... e tu che non li volevi acquistare! (IM 13)

Anche i punti esclamativi possono essere allungati per aumentare l'espressività nei messaggi imperativi, come nei seguenti ordini: ${ }^{24}$

${ }^{21}$ L'espediente è usato esclusivamente dai comunicatori (madrelingua e non) che abbiano stabilito una relazione sociale di simmetria. Dalle analisi effettuate emerge che vi ricorrono con più frequenza i parlanti nativi (5,7\% rispetto al $2 \%$ di quelli non nativi), anche perché tra gli stranieri se ne servono solo quelli con una buona padronanza dell'italiano.

22 È interessante che questa usanza si riscontri solo nei testi scritti dai madrelingua, indipendentemente dal rapporto (simmetrico/asimmetrico), e non appare mai in quelli scritti dai parlanti sloveni.

${ }^{23}$ Nella lingua parlata detta funzione pragmatica viene realizzata con la modulazione della voce.

${ }^{24}$ In base a quanto riferito dall'informatore sloveno che ha ceduto il testo, si trattava di un rapporto asimmetrico: un datore di lavoro italiano di media età che impartiva istruzioni urgenti a giovani dipendenti sloveni. 
(30) $\mathrm{K}^{* * *}$ : mail, richiesta agreement (test per 3 mesi), scambiare dettagli amministrativi, quotazioni in promo!!! [...] ricerca Firme slovene; chiamare Corte delle Firme per sapere se esistono Banche dati, e cercare su internet!!! Una volta trovate tutte le aziende di informatica, partiremo con una mail mirata al mercato sloveno!! [...] Mercoledì ore 15,00 RIUNIONE PER FARE IL PUNTO!!! [...] (IN 1)

Punti esclamativi allungati accompagnano però anche enunciati dichiarativi o esclamativi con funzione di augurio in contesti comunicativi simmetrici:

(31) Ti auguro un finesettimana leggero!!! (AP 6)

(32) Se siete in vacanza, divertitevi!!! (AP 21)

Diversamente da quanto rilevato per le e-mail d'uso comune, dove le maiuscole possono essere interpretate come l'equivalente del tono alzato (cf. Trask 2005: 67), nella posta d'affari analizzata, il ricorso al detto espediente serve a mettere in risalto l'importanza della parte evidenziata, come ad es. nel messaggio (IN 1) dell'es. (30) sopra illustrato.

Un elemento stabile della comunicazione d'affari prototipica sono le formule di apertura e di chiusura. Mentre i messaggi caratterizzati da una relativa formalità vengono iniziati da formule stereotipate riservate alla lingua scritta (Egregio signore / Gentile signore / Spettabile Ufficio), i messaggi meno formali si aprono con espressioni allocutive frequenti anche nella comunicazione faccia a faccia (cf. Fiorentino 2008: 96). Solo che, nel caso di una situazione parlata, alcune di esse (ad. es. Buongiorno / Buona sera / Buon pomeriggio / Signora + nome + buona giornata / Ciao (+ nome)) svolgono la stessa funzione di apertura, cioè per avviare una conversazione, altre invece (ad. es. Sig. + nome o soltanto Nome) possono apparire, con funzione fatica, esclusivamente al suo interno, per attirare l'attenzione dell'altro.

Simile è la situazione per quanto riguarda le formule di chiusura, dove una serie di formule ed espressioni incontrate nelle e-mail funziona alla perfezione anche nella comunicazione orale, come ad es. A più tardi. / Grazie come sempre e a presto. / A presto. / Grazie e a presto! / Grazie e buona giornata. / Grazie e buon lavoro. / Grazie, ciao. / Grazie mille. / Ciao. / Ciao e buona serata. / Ciao e a presto.

\section{CONCLUSIONE}

La comunicazione d'affari scritta, nel corso della sua istituzionalizzazione, si è sempre più allontanata dai modi tipici del "parlato", cioè della conversazione faccia a faccia (e i suoi elementi di spontaneità, oralità, deissi, referenza implicita, termini generici, assenza di elementi connettivi, modi di dire colloquiali, imprecisione, modulazione della voce, interiezioni, pause, false partenze, ecc.). Si è così arrivati alla comunicazione fortemente caratterizzata da elementi dello "scritto": modelli prototipici di lettere con macrostrutture precostituite, frasi stereotipate, atteggiamenti impersonali, modi indiretti, lessico professionale, linguaggio burocratico. Con la diffusione e il largo uso del nuovo medium, la posta elettronica, che permette una comunicazione semisincrona, si nota un riavvicinamento ai modi della conversazione 
faccia a faccia (soprattutto quando la comunicazione è di tipo informale) con il ritorno, nei messaggi scritti, di alcune caratteristiche del "parlato".

\section{Bibliografia}

ANTONELli, Giuseppe (2007) L'italiano nella società della comunicazione. Bologna: il Mulino.

BARON, Naomi (1998) "Letters by phone and speech by other means: the linguistics of Email." Language and Communication 18, 133-170.

Bazzanella, Carla (2006) "Discourse markers in Italian: Towards a 'compositional' meaning." In: K. Fisher (ed), Approaches to Discourse Markers. Amsterdam: Elsevier, 449-464.

CRYSTAL, David (2001) Language and the Internet. Cambridge: Cambridge University Press.

D'ACHILle, Paolo (2006) L'italiano contemporaneo. Bologna: il Mulino.

FASULO, Alessandra (2009) "L'epistemica debole della conversazione: quando le domande iniziano con $e$ o con ma." In: M. Fatigante/L. Mariottini/M. E. Sciubba (eds), Lingua e società. Scritti in onore di Franca Orletti. Milano: FrancoAngeli, 29-48.

Fiorentino, Giuliana (2002) "Computer-Mediated Communication: lingua e testualità nei messaggi di posta elettronica in italiano.” In: R. Bauer/H. Goebel (eds), Atti del IX congresso italo-austriaco. Wilhelmsfeld: Egert, 187-208.

Fiorentino, Giuliana (2004) "Scrivere come si parla. Variabilità diamesica e CMC: il caso dell'e-mail." Horizonte 8, 83-110.

FioRENTINO, Giuliana (2008) "Scrittura elettronica: il caso della posta elettronica." In: F. Orletti (ed), Scrittura e nuovi media. Roma: Carocci, 69-112.

LORENZETTI, Luca/Giancarlo SCHIRRU (2006) "La lingua italiana nei nuovi mezzi di comunicazione: SMS, posta elettronica e Internet." In: S. Gensini (ed), Fare comunicazione. Roma: Carocci, 71-98.

LENASSI, Nives (2010) Analiza italijanskih besedil s področja poslovne korespondence v funkciji učinkovitega tujejezičnega poučevanja ekonomistov na univerzitetni stopnji. Ljubljana: Università di Ljubljana.

Mallon, Rebecca/Charles OpPenheim (2002) "Style used in electronic mail." Aslib Proceeedings 54/1, 8-22.

Nencioni, Giovanni (1983) "Parlato-parlato, parlato-scritto, parlato-recitato." In: G. Nencioni (ed), Di scritto e di parlato. Bologna: Zanichelli, 126-179.

NidORFER ŠIšKovič, Mojca (2010) Pragmatičnojezikoslovna analiza elektronskih poslovnih pisem $v$ slovenščini. Ljubljana: Università di Ljubljana.

PeticcA, Sara (2005) "Il linguaggio delle e-mail.” In: M. Baldini/D. Marucci (eds), La parola nella galassia elettronica. Roma: Armando, 93-101.

SABatinI, Francesco (1985) "L'italiano dell'uso medio': una realtà tra le varietà linguistiche italiane.” In: G. Holtus/E. Radtke (eds), Gesprochenes Italienisch in Geschichte und Gegenwart. Tübingen: Narr, 154-184.

Trask, Robert Lawrence (2005) How to Write Effective Emails. London: Penguin. 


\section{Riassunto \\ TRATTI DEL PARLATO NELLE E-MAIL D'AFFARI IN LINGUA ITALIANA SCAMBIATE TRA PARTNER ITALIANI E SLOVENI}

Sullo sfondo delle constatazioni di vari studiosi relative alle caratteristiche dialogiche e orali nelle e-mail di natura prevalentemente privata, questa volta l'autrice ha condotto un'indagine in merito alla presenza dei tratti del parlato nella comunicazione elettronica d'affari. È stato constatato che quest'ultima, nonostante sia fortemente istituzionalizzata e perciò assai più restia all'irruzione dell'oralità nei testi, mostra pure un certo numero di tratti indubbiamente appartenenti alla comunicazione parlata. Così a livello diamesico, accanto ai messaggi formalmente assai strutturati, se ne trovano altri meno formali, fino a quelli che sembrano mimare una conversazione telefonica. A livello grafico, la punteggiatura enfatica (punti di sospensione, punti esclamativi e punti interrogativi) sembra essere usata in sostituzione della modulazione della voce (con una specifica funzione comunicativa). A livello lessicale e a livello sintattico, l'uso di alcuni verbi generici (dire e sentire) e di certe congiunzioni polifunzionali usate come segnali discorsivi ( $e$ e $m a$ ) tipici della comunicazione spontanea, allontana i messaggi dalla tradizionale formalità dell'interazione scritta. Al parlato ricordano, inoltre, anche il fenomeno sintattico della dislocazione a sinistra nonché alcune interiezioni. A livello testuale, infine, tradiscono un legame con il parlato determinate formule di apertura e di chiusura riscontrabili regolarmente in diversi punti di una conversazione faccia a faccia o in una conversazione telefonica.

Parole chiave: e-mail, parlato, istituzionalità, formalità, informalità.

\section{Povzetek \\ PRVINE GOVORNEGA V POSLOVNIH ELEKTRONSKIH SPOROČILIH, IZMENJANIH MED ITALIJANSKIMI IN SLOVENSKIMI PARTNERJI}

$\mathrm{Na}$ ozadju ugotovitev različnih raziskovalcev o elementih dialoškosti in govornega, vsebovanih v pretežno zasebnih elektronskih sporočilih, je avtorica tokrat raziskovala prisotnost lastnosti govornega $\mathrm{v}$ poslovnih e-mailih. Ugotovljeno je bilo, da čeprav le-ti zaradi svoje institucionaliziranosti dopuščajo precej manjši vdor pogovornih prvin, pa nekatere poteze $\mathrm{v}$ neformalnih besedilih vendarle izpričujejo svojo nedvomno povezanost z govorjeno komunikacijo. Tako najdemo na diamezični ravni poleg besedil, ki so formalno izrazito strukturiana, tudi besedila, ki so precej manj, vse do takih, za katere se zdi, da posnemajo telefonski razgovor. $\mathrm{Na}$ grafični ravni je raba poudarjenih ločil (tropičje, klicaji, vprašaji) prepoznana kot nadomestek za moduliranje glasu (s posebno komunikacijsko nalogo). Na leksikalni in skladenjski ravni raba nekaterih splošnopomenskih glagolov (dire /reči/ in sentire /slišati/) in nekaterih večpomenskih veznikov (uporabljenih kot diskurzivni označevalci, npr. $e$ /in/ in $m a$ /ampak, pa, a/), ki so značilni za spontano sporočanje, odmika ta sporočila od uveljavljene formalnosti pisnega sporočanja. Končno spominjajo na govorjeno tudi sintaktični pojav "premika na levo" in nekateri medmeti. Na besedilni ravni izdajajo povezanost z govorjenim diskurzom nekatere otvoritvene in zaključevalne formule, ki jih sicer redno najdemo na različnih mestih neposrednega dialoga oz. telefonskega pogovora.

Ključne besede: elektronsko sporočilo, govorno, institucionaliziranost, formalnost, neformalnost. 